\title{
FACTORING IN THE METAPLECTIC GROUP AND OPTICS
}

\author{
Marko Huhtanen AND SAntTu RuOtsalainen
}

\begin{abstract}
The metaplectic group is generated by the Fourier transform and multiplications by functions of particular exponential type. Based on the use of the metaplectic representation and a factorization of symplectic matrices, in this paper a bound on the number of terms needed to factor an arbitrary metaplectic operator is derived. The approach is constructive and numerically stable, leading to a reliable factorization algorithm in practice. The problem is partially motivated by the task of constructing lens systems in diffractive optics.
\end{abstract}

Mathematics subject classification (2010): 42B10, 78M25, 65F30. system.

Keywords and phrases: Metaplectic group, symplectic matrix, factorization, diffractive optics, lens

\section{REFERENCES}

[1] G. Frobenius, Über die mit einer Matrix vertauschbaren Matrizen, Sitzungsber. Preuss. Akad. Wiss., 1910, pp. 3-15.

[2] V. Guillemin and S. Sternberg, Symplectic Techniques in Physics, Cambridge University Press, Cambridge, 1984.

[3] G. Folland, Harmonic Analysis in Phase Space, Princeton University Press, Princeton, 1989.

[4] J. W. Goodman, Introduction to Fourier Optics, McGraw-Hill, Singapore, 1996.

[5] M. Huhtanen, Factoring matrices into the product of two matrices, BIT, 47 (2007), 793-808.

[6] P. D. LaX, Linear Algebra, John Wiley \& Sons, New York, 1997.

[7] H. TER MORSCHE AND P. OONINCX, On the integral representations for metaplectic operators, J. Fourier Anal. Appl., 8 (2002), 245-257.

[8] M. Postnikov, Lectures in Geometry, Smooth Manifolds, Mir Publishers, Moscow, 1989. 